\title{
Injection currents in lamellar crystals of gallium telluride
}

\author{
R.S. Madatov, T.B. Tagiyev, I.A. Gabulov, T.M. Abbasova \\ Azerbaijan National Academy of Sciences, Institute of Radiation Problems, 31a H.Javid avenue, Baku-143, Azerbaijan \\ Phone: +38(99412) 39 8318; fax: +38(99412) 398318
}

\begin{abstract}
The results of researches of electrical properties and injection currents in lamellar samples of p-type gallium telluride with the purpose of determination of charge transfer mechanism both in the Ohm law regime and in the range of its violation in the wide temperature interval $(77 \div 300) \mathrm{K}$ have been given. Specific resistance of the studied samples changed within $5 \cdot 10^{4} \div 6 \cdot 10^{5} \mathrm{Ohm} \cdot \mathrm{cm}$ at room temperatures. The analysis based on the Lampert theory has shown that the current-voltage characteristics at $77 \mathrm{~K}$ are in accord with monopolar injection current and one discrete level in the gallium telluride bandgap with the energy $0.18 \mathrm{eV}$ and concentration $5.6 \cdot 10^{12} \mathrm{~cm}^{-3}$. It has been found that, in the temperature range of $77 \div 300 \mathrm{~K}$ in GaTe single crystals, the mechanism of charge transfer can be ascertained with currents limited by the spatial charge.
\end{abstract}

Keywords: injection currents, lamellar crystals, electrical properties, charge transfer mechanism, temperature range, conductivity, current-voltage characteristics, bandgap.

Paper received 27.05.03; accepted for publication 17.06.03.

Electrical properties of lamellar crystals of gallium telluride have not been thoroughly investigated in comparison with other chalcogenides compounds, though its optical properties have been determined quite well $[1,2,3,4,5]$. In the works $[6,7,8]$ electrical properties of gallium telluride, such as Hall mobilities along and perpendicular to $C$ axis of crystals, the temperature dependencies of electrical conductivity, etc. have been studied. It follows from measurements of Hall mobilities that the anisotropy of electrical properties relatively to $C$ axis of crystals is higher than that of the other gallium chalcogenides.

In the work [8], injection currents in GaTe with ohmic indium contacts perpendicular to $C$ axis of crystals have been studied, and it has been shown that at room temperatures and high fields the current is determined by the double injection through a sample.

It is necessary to note that in the paper [8] the authors made their conclusions on double injection in GaTe using the only parameter - capture ratio, which significantly exceeded unity, though other characteristics of GaTe samples are identical, as in the case of monopolar injection.

In the given work, the electrical properties and injection currents in $p$-type GaTe monocrystals samples are thoroughly studied with the purpose of definition of the charges transfer mechanism both in Ohm law regime, and in the field of its violations.

Lamellar GaTe monocrystals have been grown by the Bridgman method and have $p$-type conductivity. The specific resistance of the studied samples have changed within the $5 \cdot 10^{4} \div 6 \cdot 10^{5} \mathrm{Ohm} \cdot \mathrm{cm}$ at room temperatures. In this work the results of the authors [7] have been used as data on Hall mobilities of holes perpendicular to $C$ axis, and the value of mobility parallel to $C$ axis was determined from measurements of anisotropy of the electrical conductivity by the tetra-probe Shnabel method. The values of electrical conductivity anisotropy of thick samples were used to determine the mobility parallel to $C$ axis in order to exclude a surface influence as far as possible.

The value of mobility estimated in such a way at room temperatures parallel to $C$ axis was equal to $1 \div 2 \mathrm{~cm}^{2} / \mathrm{V}$.sec. The contacts to the samples were made in vacuum from indium. The thicknesses of the studied samples were changed within $55 \div 400$ micrometers.

Volt-ampere characteristics of GaTe sample was not specially doped at different temperatures in the direction of $C$ axis has shown in Fig. 1 (curve 1, 2, 3, 4, 5). As it is seen from Fig. 1, that the volt-ampere characteristics of the samples are significantly changed with the increase 


\section{R.S. Madatov et al.: Injection currents in lamellar crystals of ...}

of temperature, i.e. if at $77 \mathrm{~K}$ temperature it is in accord with $I \sim V^{n}$, where $n=1,2,3$ and 2, 3 alternate with changing the voltage, then the dependence $I \sim V^{n}$ in double logarithmic scale deviates from a straight line at high temperatures. The analysis based on the Lampert theory has shown that the volt-ampere characteristic at $77 \mathrm{~K}$ is in accord with monopolar injection current with one discrete level in the band-gap of GaTe with $0.18 \mathrm{eV}$ energy (the other calculated parameters from Fig. 1 are given in Table 1). Table 1 shows that the calculated parameters of volt-ampere characteristics at $300 \mathrm{~K}$ are in well accord with the work data [8], though in this work the double injection in GaTe samples with indium contacts is discussed. The determination of capture ratio values both in the work [8] and from volt-ampere characteristics at $300 \mathrm{~K}$ coincide and they are equal to 36 , and at $77 \mathrm{~K}$ capture ratio value $\theta=7.57 \cdot 10^{-4}$.

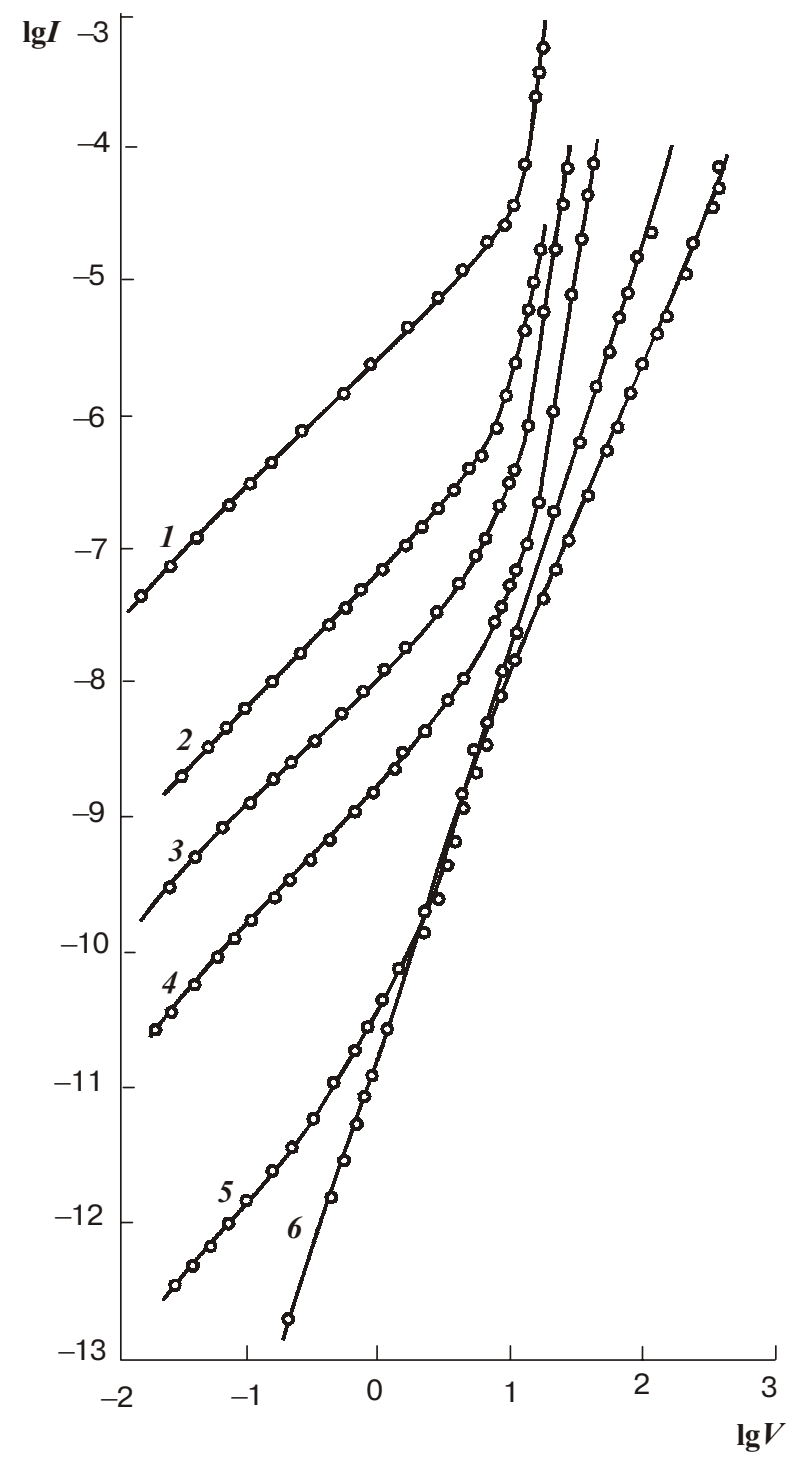

Fig. 1. Volt-ampere characteristics of GaTe sample at different temperatures $(\mathrm{K}): 1-290 ; 2-195 ; 3-184 ; 4-173 ; 5-77 ; 6-$ theoretical.

SQO, 6(3), 2003
Due to the fact that in the work [8] the temperature studies have not been conducted and besides all the conclusions on double injection were made on the basis of capture ratio and thickness dependence of current density in the field of sharp rise, we do not consider the results of this work quite correct, especially as our results on temperature dependence indicate to monopolar injection. In addition, it is known that transfer from monopolar injection to double injection with symmetrical contacts on the sample is possible only at high fields [11].

In order to prove monopolarity of injection, we have studied thickness dependencies of volt-ampere characteristics within $55 \div 400$ micrometers in the first and second quadratic sections with the change of voltage values on the sample. The exponent of current density dependence is limited by a volume charge has been determined from Fig. 2 and depending on the thickness for different samples it is equal or exceed 3.

According to Lampert's theory, such a change is possible both in the case of monopolar and double injection, if the currents are limited by the volume charge. However, special experiments on electrical luminescence and lack of section with negative differential resistance in volt-ampere characteristics indicate to flow of monopolar current in GaTe samples.

In a general case, volt-ampere characteristics of $\mathrm{GaTe}$ samples are shown in Fig. 1, and it can be presented as

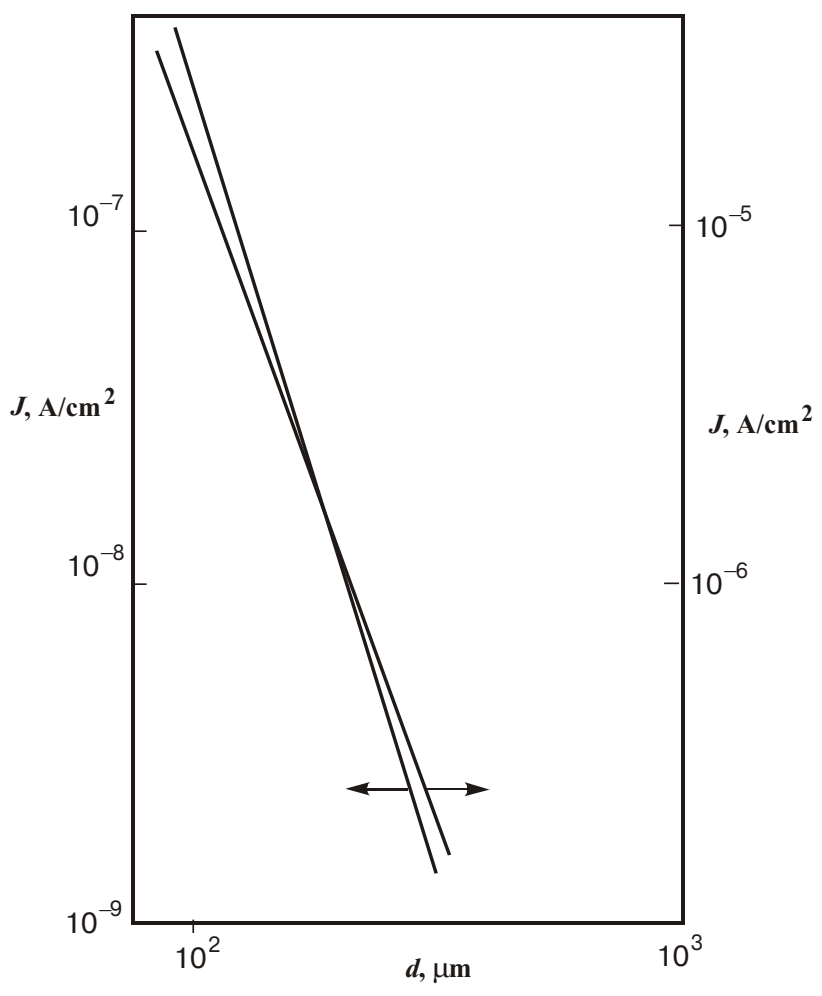

Fig. 2. Thickness dependence of current density in the first (curve 1) and the second quadratic sections (curve 2) of volt-ampere characteristics at $77 \mathrm{~K}$. 
R.S. Madatov et al.: Injection currents in lamellar crystals of ...

$I=e^{1-l} \mu_{\rho} N_{v}\left(\frac{2 l+1}{l+1}\right)^{l+1}\left(\frac{l}{l+1}\right)^{l}\left(\frac{\varepsilon \varepsilon_{o}}{N_{t}^{1}}\right)^{l} \frac{v^{l+1}}{d_{e f f}^{2 l+1}}$

where $N_{v}=1.6 \cdot 10^{19} \mathrm{~cm}^{-3}, \mu_{\rho}$ - free holes mobility, $d_{e f f}-$ effective thickness of a sample, $T_{t}$ is a characteristic temperature for exponential distribution. The formula (1) is written in the monopolar case for exponential distribution of traps in the band-gap, when $N_{t}^{1}$ is described as

$N_{t}^{1}(E)=N_{t} \exp \left(\frac{E-E_{t}}{k T_{t}}\right), E>E_{t}$

The supposed approximation $N_{t}^{1}(E)(12,13)$ is justified by the fact that there are defects in GaTe monocrystals connected with stoichiometric [2,6] and structural violations conditioned by the low energy of dislocations in the layer plane [10].

GaTe structure is referred to monoclinic syngony with special B2/m group [14]. In GaTe lattice the atoms of metal are in three crystallographically independent positions and, besides, the surroundings of all the gallium atoms in them are tetrahedral with different length of GaTe bonds. It would be natural to suppose that the levels with different energy will correspond to defects connected with lack or replacement of gallium in the band-gap. The comparison (1) with the experimental results showed a good accord in the case $N_{t}^{1}=1.2 \cdot 10^{14} \mathrm{~cm}^{-3}$ and $l=2$. Such a comparison is presented in Fig. 1 (curve 6) at $k T_{t}=0.0066 \mathrm{eV}$. This gives us opportunity to judge about the change of volt-ampere characteristics of the samples with change of temperature (Fig. 1).

Such a behavior of volt-ampere characteristics is typical in the case of exponential distribution of traps in a band-gap of semiconductor in the monopolar regime of current limited by spacial charge.

The degree of modification of volt-ampere characteristics (1) can be independently determined from the thickness dependence of current density, and it has been found that $l=2$.

The dependence $N_{t}^{1}$ from characteristic energy for exponential distribution of traps was constructed (Fig. 3) in order to find concentration of traps in a maximum of states density and to determine its energetic position in the bandgap. It was determined that the parameters $N_{t}=$ $=5.6 \cdot 10^{12} \mathrm{~cm}^{-3}$ and $E_{t}=0.18 \mathrm{eV}$. The latter value is in satisfactorily accord with the activation energy, which equals to $0.18 \mathrm{eV}$ of dark electrical conductivity of samples in the ohmic regime presented in Fig. 1 within the range $77 \div 200 \mathrm{~K}$.

It is necessary to note that the change of the Fermi quasi-level with charge accumulation and applied voltage is typical for exponential distribution of trapes in the band-gap of semiconductor

$E_{f}(v)=k T_{t} \ln \left[\frac{(l+1)^{2}}{l(l+1)} \cdot \frac{e N_{t} d_{e f f}^{2}}{\varepsilon \varepsilon_{o} v}\right]$

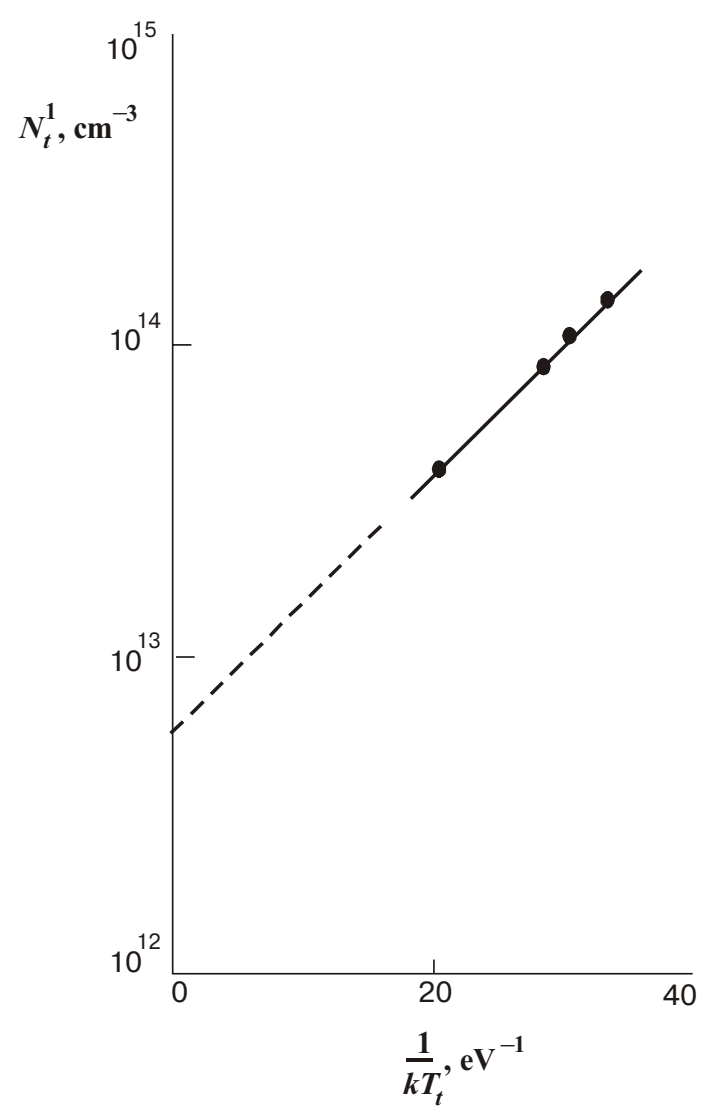

Fig. 3. Dependence $N^{1}$ on $1 / k T_{t}$ for exponential distribution of trap levels in the band-gap of the crystal.

where $E_{f}(v)$ is an averaged by the sample value $E_{f}$ that is determined by the correlation: $I=e^{\mu} N_{v} \frac{v}{d_{e f f}} \exp \left(-\frac{E_{f}}{k T}\right)$ because the field value is determined as $e=v / d_{e f f}$ is averaged by the sample.

Such dependence for GaTe samples is presented in Fig. 5, from where the area of the Fermi quasi-level change $(0.4 \div 0.18 \mathrm{eV})$ in the band-gap with charge accumulation has been determined. It is necessary to note that (1) is written for the case when distribution of traps by energies starts from a certain discrete level in the band-gap $\left(E_{t}\right)$, and not from the bottom of conduction band or a ceiling of a valence band. As we noted above, such a distribution of traps is possible in the case when there are stoichiometric violations in crystals connected with lack of cations in the crystalline lattice sites.

The quantity of total traps concentration $\left(N_{t}^{1}\right)$ in GaTe band-gap is approximately equal to $10^{14} \mathrm{~cm}^{-3}$, the concentration of traps in the maximum of state density corresponding to the energy $0.18 \mathrm{eV}$ is approximately equal to $10^{12} \mathrm{~cm}^{-3}$, temperature and field dependencies of Fermi quasi-level position allow us to assume that asymmetric distribution of levels starts from $0.18 \mathrm{eV}$, and not from the ceiling of the valence band, where states density is $10^{14} \mathrm{~cm}^{-3}$. 


\section{R.S. Madatov et al.: Injection currents in lamellar crystals of ...}

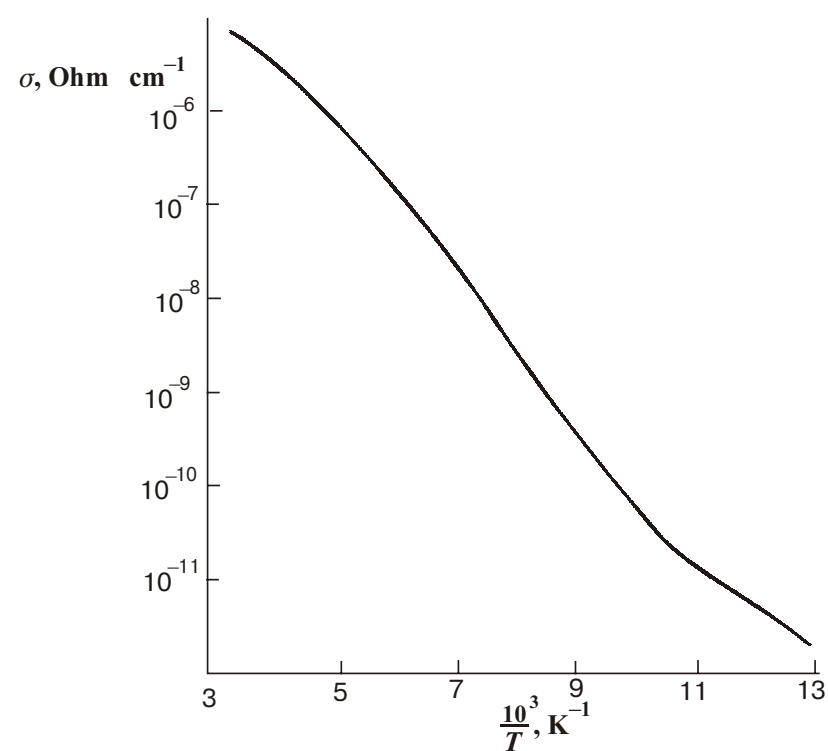

Fig. 4. Temperature dependence of electrical conductivity of GaTe sample.

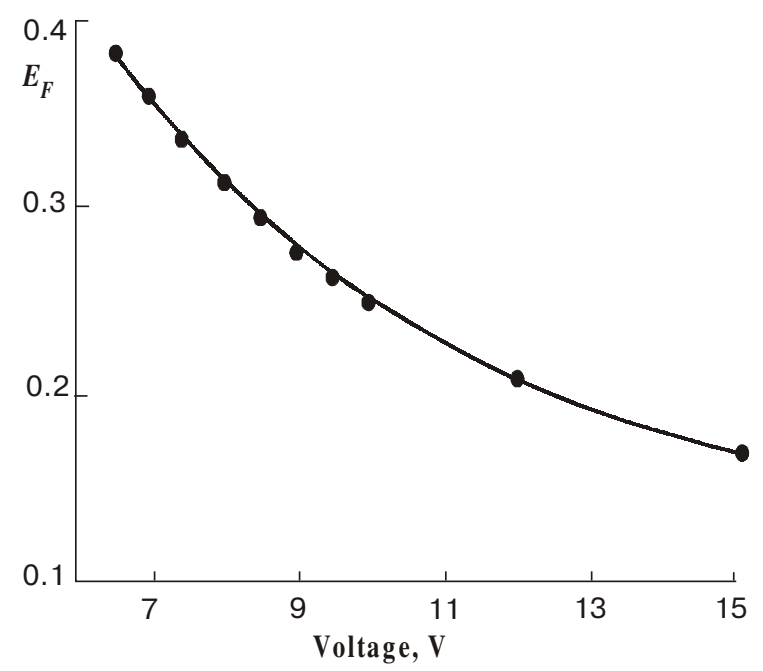

Fig. 5. Dependence of Fermi quasi-level location on the voltage applied to the sample.

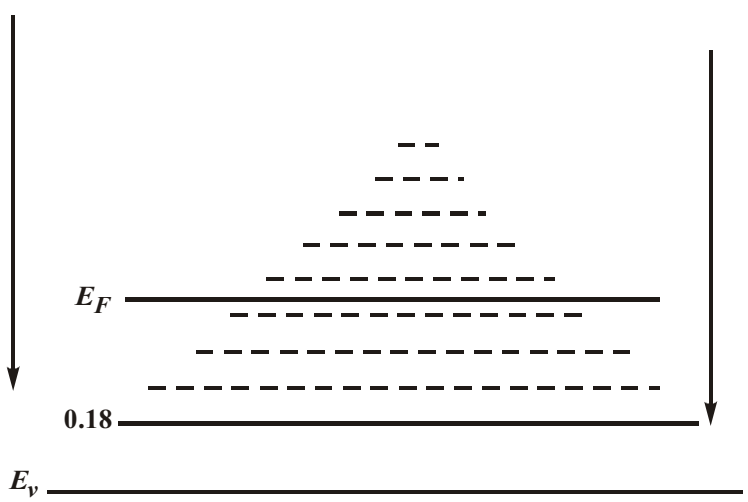

Fig. 6. Band model with one discrete level of trap and exponential distribution of traps in a band-gap of GaTe.
In literature $[2,3,6]$, the level with $0.18 \mathrm{eV}$ energy is also connected with stoichiometric violations in GaTe crystals on the basis of studies of galvanomagnetic $(0.152 \mathrm{eV})$ and photoelectric $(0.165 \mathrm{eV})$ properties. Taking into account the above-mentioned experimental results, the band model for GaTe with one trap level and exponential distribution of traps in a band-gap can be presented as in Fig. 6. The suggested model well explains the experimental results obtained by the method of measurements of currents limited by the volume charge and temperature dependences of electrical conductivity in GaTe monocrystal samples.

\section{References}

1. L.N. Kurbatov, A.I. Dirochka, B.A. Sosin, Temperature dependence of photocurrent and of period of relaxation of gallium monotelluride photoconductivity // FTP, 17(1), pp. 176-179 (1983).

2. L.N. Kurbatov, A.I. Dirochka, B.A. Sosin, Spectrum of gallium monotelluride photoconductivity // FTP, 16(9), pp. 16441646 (1982).

3. L.N. Kurbatov, A.I. Dirochka, B.A. Sosin, Impact of exciton polaritons on GaSe absorption brink // FTP, 13(1), pp. 75-82 (1979).

4. M. Grandolfo, E. Gratton, F. Anfosso Somma, P. Veccha // Phys. St. Sol. (b), 48(2), pp. 729-735 (1971).

5. J.L. Brebner, G. Sisher, E. Mosser // J. Phys. Chem. Sol., 23, pp. 1417-1421 (1962).

6. C. Manfredotti, R. Murri, A. Rizzo, L. Vasanelli, G. Micossi // Phys. St. Sol. (a), 29(2), pp. 475-480 (1975).

7. G. Fisher, J.L. Brebner // J. Phys. Chem. Sol. 23, pp. 13631370 (1962).

8. C. De Blasi, S. Golassini, C. Manfregotti, G. Micossi, A. Topore // Lettere Al Nuovo Cimento 23(10), pp. 395-400 (1978).

9. P. Schabell // Leits Angero Phys. 22, pp. 136-146 (1967).

10. V.B. Sapre, C. Made // J. Phys.Chem. Sol. 34, pp. 1351-1356 (1975).

11. M. Lampert, P. Mark, Injection currents in solids. M., 1973, pp. 416.

12. P.J. Reucroft, F.D. Mullins // J.Phys. Chem. Sol. 35, pp. 347353 (1974).

13. M. Schadt, D.F. Williams // J.Phys. 50(10), pp. 4364-4368 (1969).

14. M. Julien-Pouzol, S. Jaulmes, M. Guittard, F. Alapini // Acta Gryst. B35, pp. 2848-2851 (1979). 